\title{
Timing of intervention in high-risk non-ST-elevation acute coronary syndromes in PCI versus non-PCI centres
}

\author{
Sub-group analysis of the ELISA-3 trial
}

\author{
E.A. Badings ${ }^{1} \cdot$ W.S. Remkes ${ }^{2} \cdot$ J-H.E. Dambrink ${ }^{2} \cdot$ S.H.K. The ${ }^{3} \cdot$ \\ J. Van Wijngaarden ${ }^{1} \cdot$ G. Tjeerdsma ${ }^{4}$ S. Rasoul ${ }^{5,6} \cdot$ J.R. Timmer ${ }^{2}$. \\ M.L.J. van der Wielen ${ }^{3}$ - D.J.A. Lok ${ }^{1}$ A.W.J. van 't Hof ${ }^{2}$ \\ Published online: 28 January 2016 \\ (C) The Author(s) 2016. This article is published with open access at Springerlink.com
}

\begin{abstract}
Aims To compare the effect of timing of intervention in patients with non-ST-elevation acute coronary syndrome (NSTE-ACS) in percutaneous coronary intervention (PCI) versus non-PCI centres.

Methods and results A post-hoc sub-analysis was performed of the ELISA III trial, a randomised multicentre trial investigating outcome of early $(<12 \mathrm{~h})$ versus late $(>48 \mathrm{~h})$ angiography and revascularisation in 542 patients with highrisk NSTE-ACS. 90 patients were randomised in non-PCI centres and tended to benefit more from an early invasive strategy than patients included in the PCI centre (relative risk 0.23 vs. 0.85 [ $p$ for interaction $=0.089$ ] for incidence of the combined primary endpoint of death, reinfarction and recurrent ischaemia after 30 days of follow-up). This was largely driven by reduction in recurrent ischaemia. In nonPCI centres, patients randomised to the late group had a 4 and 7 day longer period until PCI or coronary artery bypass grafting, respectively. This difference was less pronounced in the PCI centre.
\end{abstract}

\footnotetext{
A.W.J. van 't Hof

a.w.j.vant.hof@isala.nl

Deventer Hospital, Deventer, The Netherlands

2 Isala Klinieken, Dokter van Heesweg 2, 8025AB Zwolle, The Netherlands

3 Treant Zorggroep location Bethesda, Hoogeveen, The Netherlands

4 Hospital De Tjongerschans, Heerenveen, The Netherlands

5 Atrium Medisch Centrum, Heerlen, The Netherlands

6 Maastricht UMC, Maastricht, The Netherlands
}

Conclusions This post-hoc analysis from the ELISA-3 trial suggests that NSTE-ACS patients initially hospitalised in non-PCI centres show the largest benefit from early angiography and revascularisation, associated with a shorter waiting time to revascularisation. Improved patient logistics and transfer between non-PCI and PCI centres might therefore result in better clinical outcome.

Keywords Non ST-segment elevation acute coronary syndrome $\cdot$ Revascularisation · Timing · Elderly $\cdot$ Clinical outcome $\cdot$ Interventional clinics · Non-interventional clinics

\section{Introduction}

A routinely invasive strategy (angiography and revascularisation if applicable) is recommended by the current guidelines $[1,2]$ in high-risk patients with non-ST-elevation acute coronary syndrome (NSTE-ACS). The majority of trials and meta-analysis on this topic showed a reduction in the incidence of cardiovascular death and myocardial infarction (MI) in the medium to long term [3-9], although for example the Dutch Ictus study (Invasive versus Conservative Treatment in Unstable Coronary Syndromes [10]) did not. Studies investigating optimal timing of intervention showed conflicting results. Meta-analysis of randomised controlled trials (RCTs) [11, 12] and observational studies [13] comparing early to later timing showed that early timing of intervention does not lead to a reduction in the incidence of death or myocardial infarction but at most reduces the incidence of recurrent ischaemia.

A limitation of these studies is that they were largely conducted in tertiary care, high-volume, percutaneous coronary intervention (PCI) centres. In the Netherlands, as in many 
other European countries, however, a considerable number of these patients are initially hospitalised in non-PCI centres. After medical stabilisation and coronary angiography, the patient is then transferred to the PCI centre for revascularisation, if needed. This makes generalisability of the study results to clinical practice questionable.

Observational studies $[14,15]$ showed that patients admitted to a centre with angiography and PCI facilities were more likely to receive an invasive strategy, had a lower risk for refractory or recurrent angina at the cost of higher risk for stroke and major bleeding. No difference was found in the incidence of cardiovascular death, myocardial infarction or stroke at 6 months. According to the investigators, these findings support the strategy of directing patients with suspected ACS to the nearest hospital with acute care, irrespective of the availability of interventional facilities. Because these studies were non-randomised and performed about 15 years ago, when background medication was different from today, these data should be interpreted with caution.

The ELISA 3 trial compared an early versus delayed invasive strategy in a population of high-risk patients hospitalised for NSTE-ACS in 6 centres ( 5 non-PCI and 1 PCI) in the Netherlands. The aim of the current sub-study is to investigate the clinical effect of timing of invasive strategy in patients randomised in the participating non-PCI centres and to compare this to patients randomised in the PCI centre.

\section{Methods}

The ELISA-3 study was an investigator-initiated, randomised, open, multicentre study. Rationale, design and results have been published previously [16]. Patients were eligible if they were hospitalised with ischaemic chest pain or dyspnoea at rest and had at least 2 out of 3 of the following high-risk characteristics: (1) evidence of extensive myocardial ischaemia on ECG (shown by new cumulative ST depression $>5 \mathrm{~mm}$ or temporary ST-segment elevation in 2 contiguous leads $<30 \mathrm{~min}$ ), (2) elevated biomarkers (tropo$\operatorname{nin} \mathrm{T}>0.10 \mu \mathrm{g} / \mathrm{l}$ or myoglobin $>150 \mu \mathrm{g} / \mathrm{l})$ or elevated creatine kinase-myocardial band (CK-MB) fraction $(>6 \%$ of total (CK) and (3) age above 65 years. Randomisation had to take place within $24 \mathrm{~h}$ of the last episode of ischaemic symptoms. Exclusion criteria were persistent ST-segment elevation, symptoms of ongoing myocardial ischaemia despite optimal medical therapy, contraindication for diagnostic angiography, active bleeding, cardiogenic shock, acute posterior infarction and life expectancy less than 1 year. The trial was conducted in six Dutch hospitals of which one had 24-hour facilities for (primary) PCI and coronary artery bypass grafting (CABG). The study was registered in the ISRCTN Register (ISRCTN39230163).

\section{Patients}

After giving written informed consent, patients were randomly assigned to an immediate or delayed treatment strategy. In patients assigned to the immediate treatment strategy, angiography was performed as soon as possible but within $12 \mathrm{~h}$ of randomisation. Patients assigned to the delayed treatment strategy underwent angiography no sooner than $48 \mathrm{~h}$ after randomisation unless, despite optimal medical therapy, clinical instability or recurrent ischaemia warranted emergency angiography. Patients recruited at a non-PCI centre and randomised to the immediate intervention group were urgently transferred to the PCI centre for angiography and subsequent revascularisation; in case of assignment to delayed intervention, angiography was performed at the non-PCI centre and the patient was transferred to the PCI centre for intervention if necessary. All patients were treated according to the current guidelines.

\section{Endpoints}

Primary endpoint of the ELISA-3 study as well for the current sub-analysis was the combined incidence of all-cause mortality, re-infarction and/or recurrent ischaemia at 30-day follow-up. Secondary endpoints were enzymatic infarct size as assessed by a single cardiac troponin T, at $72-96 \mathrm{~h}$ after admission or at discharge and the percentage of patients without a rise in CK-MB during admission. In addition, bleeding complications were assessed. Major bleeding was defined as bleeding with a haemoglobin drop of $\geq 2 \mathrm{mmol} / 1$ or a blood transfusion of 2 or more units. All endpoints were adjudicated by an independent endpoint committee.

\section{Statistical analysis}

Data were analysed according to the intention-to-treat analysis. Continuous variables were expressed as median and interquartile range (IQR) and were compared between the intervention groups using a Mann-Whitney U test. Categorical data were described by proportions and compared with the Chi square or Fisher's exact test. Logistic regression was used to calculate the $p$-value of the interaction between the effect of the intervention and the subgroups upon the primary endpoint. All tests were two-sided and an alpha of $5 \%$ was used. Statistical analysis was performed with SPSS (version 20).

\section{Results}

\section{Comparison of PCI vs. non-PCI centres}

Of the total number of 542 patients in the trial, $444(82 \%)$ were included in the PCI centre and 90 in one of the 5 non- 
PCI centres. Eight patients were excluded, 5 for withdrawn consent and 3 for major protocol violations. Baseline characteristics and clinical outcome are shown in Table 1. Patients in non-PCI centres were older and there were less smokers. Time from admission to randomisation was comparable. Time from randomisation to start of angiography was longer in the non-PCI centres ( 23.8 vs. $15.1 \mathrm{~h}, p=0.07)$ as was time from angiography to PCI $(0.75$ vs. $0.37 \mathrm{~h}, p<0.001)$ and angiography to CABG (122.8 vs. $189.9 \mathrm{~h}, p=0.037)$. No difference was found in the incidence of the primary endpoint between the two groups or in the individual components. The percentage of patients without a rise in CK-MB during admission was significantly higher in patients from the non-PCI centres (52.6 vs. $33.1 \%, p=0.01)$. Incidence of bleeding events was comparable.

\section{Effect of early vs. delayed treatment in patients included in non-PCI centres}

Of the 90 patients who were included in a non-PCI centre, $45(50 \%)$ were randomised to immediate treatment and 45 to a delayed invasive treatment strategy. Baseline characteristics were largely comparable (Table 2), with exception of gender and percentage of smokers. As intended by the protocol, median time from randomisation to angiography was $3.0 \mathrm{~h}$ in the immediate and $70 \mathrm{~h}$ in the delayed treatment group. In addition, time from the start of the coronary angiography to revascularisation (PCI or CABG, if any) was 0.7 vs. $146 \mathrm{~h}$; for PCI 0.37 vs. $96.5 \mathrm{~h}$ [4.0 days] and for CABG 115 [4.8 days] vs. $275 \mathrm{~h}$ [11.5 days]. The combined primary endpoint occurred in $4.7 \%$ of the patients in the immediate treatment group and $20.5 \%$ of the patients in the delayed treatment group $(p=0.027)$. This difference was driven by a reduction in the occurrence of recurrent ischaemia in the immediately treated patients ( 2.3 vs. $18.2 \%, p=0.03$ in the immediate and delayed treatment group respectively).The incidence of the secondary endpoints and bleeding events did not differ.

\section{Effect of timing of intervention in patients from PCI centre vs. non-PCI centres}

The effect of the timing of the intervention on the primary endpoint is shown in Fig. 1. Patients included in a non-PCI centre tended to benefit more from an early invasive strategy than those included in the PCI centre (relative risk 0.23 vs. 0.85$)$ but this difference was not statistically significant $(p$-value for interaction $=0.089)$ and largely driven by reduction in recurrent ischaemia. No differences were found in the incidence of secondary endpoints and bleeding.

\section{Discussion}

This sub-study, comparing the effect of timing of intervention in high-risk non-ST-elevation acute coronary syndromes between patients hospitalised in PCI and non-PCI centres shows that non-PCI centre patients tend to benefit more from an early invasive strategy, mainly due to reduction in recurrent ischaemia. These results were found in relationship to a much larger difference in revascularisation times between early and late treated patients in the patients admitted primarily in a non-PCI centre.

In patients initially hospitalised in non-PCI centres, median time from coronary angiography to revascularisation was longer in those randomised to a delayed strategy compared with an early invasive strategy (Fig. 2). This was caused by a difference in patient logistics: patients randomised to an immediate strategy were urgently transferred to the PCI centre where the coronary angiography andwhen appropriate - in the same procedure a PCI was performed. Patients randomised to a delayed invasive strategy underwent a coronary angiography in the non-PCI centre and underwent a PCI later on, after their angiogram had been discussed with the heart team consisting of interventional cardiologists and thoracic surgeons, and transfer to the PCI centre. This resulted in an additional waiting time after angiography of 4 days for PCI and even 7 days for CABG. For patients randomised in the PCI centre, this difference did not occur, because all patients underwent coronary angiography and PCI (if feasible) in the same procedure, irrespective of the group they were randomised to. Median waiting time for $\mathrm{CABG}$ in patients hospitalised in the PCI centre was $123 \mathrm{~h}$ (5.1 days), comparable with patients randomised to the early invasive strategy in non-PCI centres.

This difference in logistics of patients with NSTE-ACS between PCI and non-PCI centres also exists in daily practice. In PCI centres, angiography and revascularisation are generally performed in the same procedure, while patients in non-PCI centres first undergo a coronary angiography, then are discussed with the team of the PCI centre and are scheduled for revascularisation a couple of days later. In the meantime, these patients are prone to developing recurrent ischaemic symptoms, as can been seen in the Kaplan-Meyer curve of this group of patients for event-free survival from recurrent ischaemia in the first week (Fig. 3). Considering this, shortening the time between angiography and revascularisation might reduce the recurrence of ischaemia and improve clinical outcome in patients initially hospitalised in a non-PCI centre. Agreements with PCI centres to assure short access time for revascularisation and application of information technologies for fast distant evaluation of angiograms may be helpful. Periodic national audits, as for example snapshot registries of the National Cardiovascular Data Registry (NCDR), could be used to monitor the 
Table 1 Baseline characteristics, study endpoints and clinical outcomes of patients randomised in PCI versus non-PCI centres

\begin{tabular}{|c|c|c|c|c|}
\hline & & PCI centre $(n=444)$ & Non-PCI centres $(n=90)$ & $P$-value \\
\hline \multirow[t]{2}{*}{ Demographics } & Age (years, IQR) & $71.19(62.9-78.26)$ & $73.51(67.84-78.41)$ & 0.017 \\
\hline & Male gender, $n(\%)$ & $302 / 444(68.0 \%)$ & $59 / 90(65.6 \%)$ & 0.649 \\
\hline GRACE score & (median, IQR) & $136(117-154)$ & $135(119-151)$ & 0.877 \\
\hline \multirow[t]{8}{*}{ Medical history $(n, \%)$} & Hypertension & $248 / 444(55.9 \%)$ & $52 / 90(57.8 \%)$ & 0.738 \\
\hline & Smoking & $113 / 444(25.5 \%)$ & $14 / 90(15.6 \%)$ & 0.044 \\
\hline & Diabetes & $102 / 444(23.0 \%)$ & $16 / 90(17.8 \%)$ & 0.279 \\
\hline & Previous MI & $84 / 444(18.9 \%)$ & $16 / 90(17.8 \%)$ & 0.800 \\
\hline & Previous TIA & $22 / 444(5.0 \%)$ & $6 / 90(6.7 \%)$ & 0.447 \\
\hline & Previous stroke & $20 / 444(4.5 \%)$ & $1 / 90(1.1 \%)$ & 0.228 \\
\hline & Previous PCI & $89 / 444(20.0 \%)$ & $15 / 90(16.7 \%)$ & 0.461 \\
\hline & Previous CABG & $59 / 444(13.3 \%)$ & $10 / 90(11.1 \%)$ & 0.574 \\
\hline \multirow[t]{5}{*}{ Time (hours, median, IQR) } & Admission-randomisation & $2.05(1.11-4.11)$ & $2.16(0.08-6.36)$ & 0.910 \\
\hline & Randomisation angiography & $15.13(2.29-54.07)$ & $23.83(2.83-70.05)$ & 0.070 \\
\hline & Angiography-PCI & $0.37(0.18-0.65)$ & $0.75(0.30-93.25)$ & $<0.001$ \\
\hline & Angiography - CABG & $122.78(49.93-230.85)$ & $189.92(96.78-325.27)$ & 0.037 \\
\hline & Angiography-revascularisation & $0.47(0.23-43.66)$ & $69.17(0.43-190.45)$ & $<0.001$ \\
\hline \multirow[t]{3}{*}{ Extent CAD } & One vessel & $113 / 434(26.0 \%)$ & $27 / 86(31.4 \%)$ & 0.494 \\
\hline & Two vessel & $144 / 434(23.2 \%)$ & $24 / 86(27.9 \%)$ & \\
\hline & Three vessel & $127 / 434(29.3 \%)$ & $28 / 86(32.6 \%)$ & \\
\hline \multirow[t]{5}{*}{ Infarct-related artery } & LAD & $133 / 382(34.8 \%)$ & $34 / 79(43.0 \%)$ & 0.340 \\
\hline & RCA & $81 / 382(21.2 \%)$ & $15 / 79(19.0 \%)$ & \\
\hline & Circumflex & $90 / 382(23.6 \%)$ & $22 / 79(23.6 \%)$ & \\
\hline & Left main & $7 / 382(1.8 \%)$ & $1 / 79(1.3 \%)$ & \\
\hline & Graft & $34 / 382(8.9 \%)$ & $4 / 79(5.1 \%)$ & \\
\hline \multirow[t]{3}{*}{ Treatment } & PCI & $254 / 384(66.1 \%)$ & $44 / 79(55.7 \%)$ & 0.140 \\
\hline & $\mathrm{CABG}$ & $87 / 384(22.7 \%)$ & $26 / 79(32.9 \%)$ & \\
\hline & Medical & $43 / 384(11.2 \%)$ & 9/79 (11.4\%) & \\
\hline \multirow[t]{4}{*}{ Primary endpoint (\%) } & Combined endpoint ${ }^{a}$ & $52 / 436(11.9 \%)$ & $11 / 87(12.6 \%)$ & 0.851 \\
\hline & Death & $5 / 436(1.1 \%)$ & $1 / 87(1.1 \%)$ & $>0.99$ \\
\hline & MI & $5 / 436(1.1 \%)$ & $2 / 87(2.3 \%)$ & 0.329 \\
\hline & Recurrent ischaemia & $44 / 436(10.1 \%)$ & 9/87 (10.3\%) & 0.943 \\
\hline \multirow[t]{2}{*}{ Secondary endpoints } & Enzymatic infarct size ${ }^{\mathrm{b}}$ & $0.31(0.11-0.86)$ & $0.30(0.11-0.73)$ & 0.918 \\
\hline & $\%$ patients without $\mathrm{CKMB}$ rise & $147 / 444(33.1 \%)$ & $40 / 76(52.6 \%)$ & 0.001 \\
\hline \multirow[t]{4}{*}{ Bleeding } & Any bleeding & $93 / 436(21.3 \%)$ & $19 / 87(21.8 \%)$ & 0.916 \\
\hline & Major bleeding & $52 / 436(11.9 \%)$ & $8 / 87(9.2 \%)$ & 0.465 \\
\hline & CABG-related bleeding & $79 / 436(18.1 \%)$ & $14 / 87(16.1 \%)$ & 0.652 \\
\hline & CABG-related major bleeding & $47 / 436(10.8 \%)$ & $7 / 87(8.0 \%)$ & 0.444 \\
\hline
\end{tabular}

$C A B G$ coronary artery bypass graft, $C A D$ coronary artery disease, $C K M B$ creatine kinase-MB, $I Q R$ inter quartile range, $L A D$ left anterior descending, $M I$ myocardial infarction, $P C I$ percutaneous coronary intervention, $R C A$ right coronary artery, $T I A$ transient ischaemic attack.

${ }^{a}$ Combined primary endpoint $=$ incidence of death, reinfarction and recurrent ischaemia at 30-day follow-up.

bingle troponin T 72-96 h after admission ( $\mu \mathrm{g} / 1$, median, IQR).

achievement of pre-determined quality indicators. Because the role of the ECG in initial ACS triage is limited [17], other factors should also be taken into account with the assessment of urgency for revascularisation.

As far as we know, the ELISA 3 trial is the first randomised trial investigating the effect of timing of intervention in high-risk NSTE-ACS patients that included patients in both PCI and non-PCI centres, giving the opportunity to compare outcome in these two types of centres. No significant differences in clinical outcome were found, showing that initial medical stabilisation and angiography in nonPCI centres is safe and feasible.

This study has the following limitations. Clinical characteristics of both patient groups differ. For example, slightly more patients in the PCI centre had no significant coronary artery disease (11.5 vs. $8.1 \%$ ) which might have influenced 
Table 2 Baseline characteristics, study endpoints and clinical outcome of patients randomised in non-PCI centres

\begin{tabular}{|c|c|c|c|c|}
\hline & & Early treatment $(n=45)$ & Late treatment $(n=45)$ & $P$-value \\
\hline \multirow[t]{2}{*}{ Demographics } & Age (years, IQR) & $73.78(69.97-78.41)$ & $72.97(67.55-77.97)$ & 0.377 \\
\hline & Male gender, $n(\%)$ & $35 / 45(77.8 \%)$ & $24 / 45(53.3 \%)$ & 0.015 \\
\hline GRACE score & (median, IQR) & $136(129-157)$ & $130(113-149)$ & 0.406 \\
\hline \multirow[t]{8}{*}{ Medical history $(n, \%)$} & Hypertension & $24 / 45(53.3 \%)$ & $28 / 45(62.2 \%)$ & 0.393 \\
\hline & Smoking & $3 / 45(6.7 \%)$ & $11 / 45(24.4 \%)$ & 0.020 \\
\hline & Diabetes & $6 / 45(13.3 \%)$ & $10 / 45(22.2 \%)$ & 0.270 \\
\hline & Previous MI & $5 / 45(11.1 \%)$ & $11 / 45(24.4 \%)$ & 0.098 \\
\hline & Previous TIA & $3 / 45(6.7 \%)$ & $3 / 45(6.7 \%)$ & $>0.99$ \\
\hline & Previous stroke & $0 / 45(0.0 \%)$ & $1 / 45(2.2 \%)$ & $>0.99$ \\
\hline & Previous PCI & $6 / 45(13.3 \%)$ & $9 / 45(20.0 \%)$ & 0.396 \\
\hline & Previous $\mathrm{CABG}$ & $6 / 39(13.3 \%)$ & $4 / 45(8.9 \%)$ & 0.502 \\
\hline \multirow[t]{5}{*}{ Time (hours, median, IQR) } & Admission - randomisation & $2.8(0.08-5.84)$ & $1.87(0.06-9.06)$ & 0.994 \\
\hline & Randomisation - angiography & $3.04(2.23-4.03)$ & $70.05(50.6-112.58)$ & $<0.001$ \\
\hline & Angiography - PCI & $0.37(0.24-0.73)$ & $96.52(29-146.28)$ & $<0.001$ \\
\hline & Angiography - CABG & $114.75(42.05-219.53)$ & $275.25(175.57-494.15)$ & 0.012 \\
\hline & Angiography-revascularisation & $0.71(0.29-63.69)$ & $146.28(82.04-296.96)$ & $<0.001$ \\
\hline \multirow[t]{3}{*}{ Extent CAD } & One vessel & $15 / 44(34.1 \%)$ & $12 / 42(28.6 \%)$ & 0.531 \\
\hline & Two vessel & $10 / 44(22.7 \%)$ & $14 / 42(33.3 \%)$ & \\
\hline & Three vessel & $14 / 44(31.8 \%)$ & $14 / 42(33.3 \%)$ & \\
\hline \multirow[t]{5}{*}{ Infarct-related artery } & LAD & $18 / 39(46.2 \%)$ & $16 / 40(40.0 \%)$ & 0.461 \\
\hline & RCA & $7 / 39(17.9 \%)$ & $8 / 40(20.0 \%)$ & \\
\hline & Circumflex & $9 / 39(23.1 \%)$ & $13 / 40(32.5 \%)$ & \\
\hline & Left main & $0 / 39$ & $1 / 40(2.5 \%)$ & \\
\hline & Graft & $2 / 39(5.1 \%)$ & $2 / 40(5.0 \%)$ & \\
\hline \multirow[t]{3}{*}{ Treatment } & PCI & $24 / 39(61.5 \%)$ & $20 / 40(50.0 \%)$ & 0.685 \\
\hline & CABG & $11 / 39(28.2 \%)$ & $15 / 40(37.5 \%)$ & \\
\hline & Medical & $4 / 39(10.3 \%)$ & $5 / 40(12.5 \%)$ & \\
\hline \multirow[t]{4}{*}{ Primary endpoint (\%) } & Combined endpoint ${ }^{a}$ & $2 / 43(4.7 \%)$ & $9 / 44(20.5 \%)$ & 0.027 \\
\hline & Death & $0 / 43(0.0 \%)$ & $1 / 44(2.3 \%)$ & $>0.99$ \\
\hline & MI & $1 / 43(2.3 \%)$ & $1 / 44(2.3 \%)$ & $>0.99$ \\
\hline & Recurrent ischaemia & $1 / 43(2.3 \%)$ & $8 / 44(18.2 \%)$ & 0.030 \\
\hline \multirow[t]{2}{*}{ Secondary endpoints (\%) } & Enzymatic infarct size ${ }^{\mathrm{b}}$ & $0.36(0.13-0.73)$ & $0.29(0.05-1.10)$ & 0.538 \\
\hline & $\%$ patients without CKMB rise & $22 / 44(50.0 \%)$ & $18 / 32(56.3 \%)$ & 0.590 \\
\hline \multirow[t]{4}{*}{ Bleeding } & Any bleeding & $9 / 43(20.9 \%)$ & $10 / 44(22.7 \%)$ & 0.839 \\
\hline & Major bleeding & $4 / 43(9.3 \%)$ & $4 / 44(9.1 \%)$ & $>0.99$ \\
\hline & CABG-related bleeding & $6 / 43(14.0 \%)$ & $8 / 44(18.2 \%)$ & 0.592 \\
\hline & CABG-related major bleeding & $3 / 43(7.0 \%)$ & $4 / 44(9.1 \%)$ & $>0.99$ \\
\hline
\end{tabular}

$C A B G$ coronary artery bypass graft, $C A D$ coronary artery disease, $C K M B$ creatine kinase-MB, IQR inter quartile range, $L A D$ left anterior descending, $M I$ myocardial infarction, $P C I$ percutaneous coronary intervention, $R C A$ right coronary artery, $T I A$ transient ischaemic attack.

${ }^{a}$ Combined primary endpoint $=$ incidence of death, reinfarction and recurrent ischaemia at 30 day follow-up.

${ }^{\mathrm{b}}$ Single troponin $\mathrm{T}$ 72-96 $\mathrm{h}$ after admission ( $\mu \mathrm{g} / 1$, median, IQR).

our findings. Further, this sub-study is based on a posthoc sub-group analysis with a relatively small number of patients. Therefore, our findings should be interpreted with caution. From our data, the cause of the trend towards a higher risk of recurrent ischaemia in patients randomised to delayed invasive strategy in non-PCI clinics cannot be proven. Therefore, our hypothesis that this might be caused by a longer time interval between angiography and revascularisation needs further investigation. If new studies were to confirm our hypothesis, a change in the management of this large group of patients might be considered.

The main finding of the ELISA-3 study was that in highrisk NSTE-ACS patients, early angiography and revascularisation led to a non-significant $30 \%$ relative risk reduction 


$\begin{array}{llrlr} & \text { Number (\%) } & \text { Total } & \text { Number }(\%) & \text { Tota } \\ \text { Primary endpoint } & 28(12.9 \%) & 217 & 24(11.0 \%) & 219 \\ \text { PCl centre } & 9(20.5 \%) & 44 & 2(4.7 \%) & 43 \\ \text { Non PCl centres } & & & & \\ \text { Mortality } & 2(0.9 \%) & 217 & 3(1.4 \%) & 219 \\ \mathrm{PCl} \text { centre } & 1(2.3 \%) & 44 & 0(0.0 \%) & 43 \\ \mathrm{Non} \mathrm{PCl} \text { centres } & & & & \\ \mathrm{MI} & 1(0.5 \%) & 217 & 4(1.8 \%) & 219 \\ \mathrm{PCl} \text { centre } & 1(2.3 \%) & 44 & 1(2.3 \%) & 43 \\ \text { Non PCl centres } & & & & \\ \text { Recurrent Ischaemia } & 25(11.5 \%) & 217 & 19(8.7 \%) & 219 \\ \mathrm{PCl} \text { centre } & 8(18.2 \%) & 44 & 1(2.3 \%) & 43 \\ \text { Non PCl centres } & & & \end{array}$

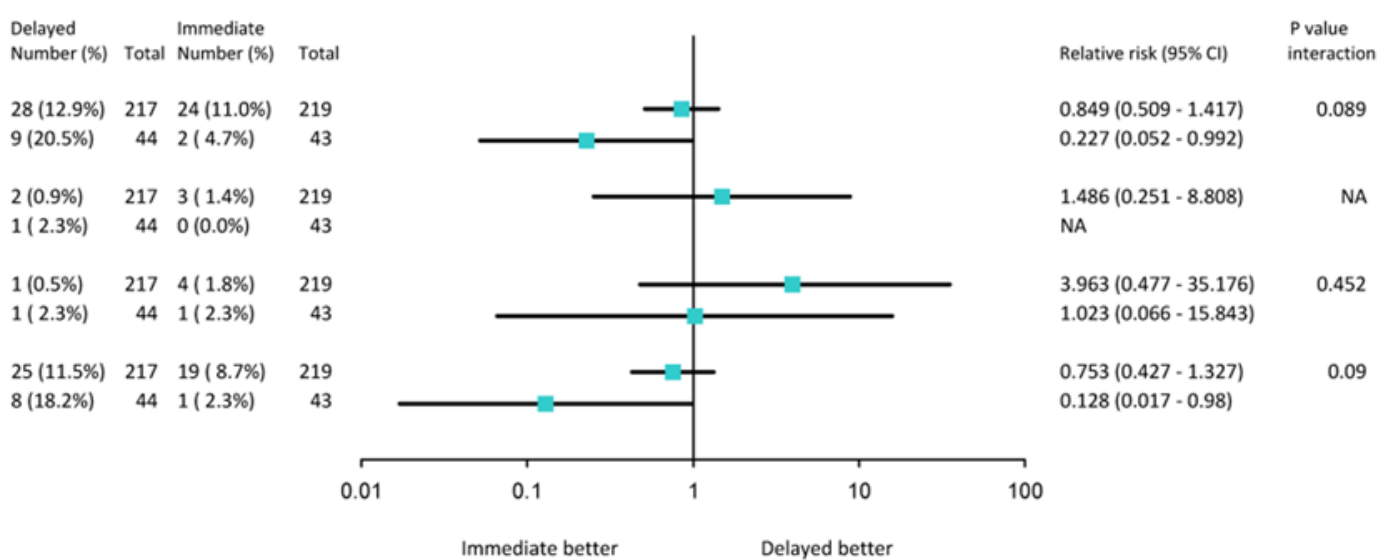

Fig. 1 Forest plot of relative risk of primary and secondary endpoints at 30 days of follow-up in patients randomised to early or late intervention in a PCI centre versus non-PCI centres. Data are numbers or per- centages, unless otherwise indicated. Percentages are number of events divided by number of patients. Squares and horizontal bars represent within-subgroup relative-risk and $95 \%$ CIs, respectively, on a log scale

Fig. 2 Timing of procedures in PCI and non-PCI-centres for patients randomised to early or late intervention. Hosp hospitalisation, random randomisation, $C A G$ coronary angiography, $P C I$ percutaneous coronary intervention

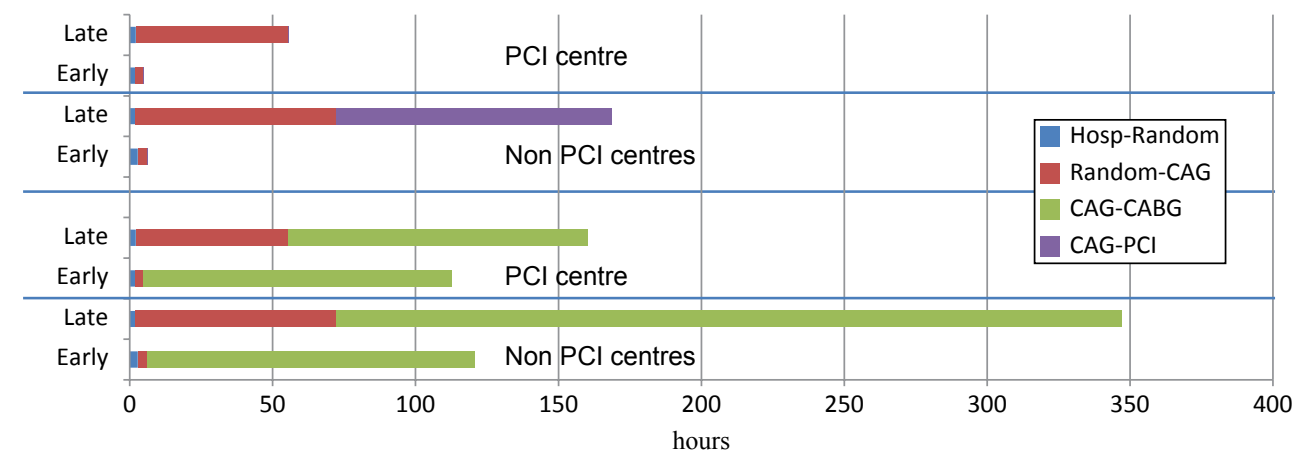

Fig. 3 Kaplan-Meyer curves for event-free survival from recurrent ischaemia for each of the 4 treatment groups

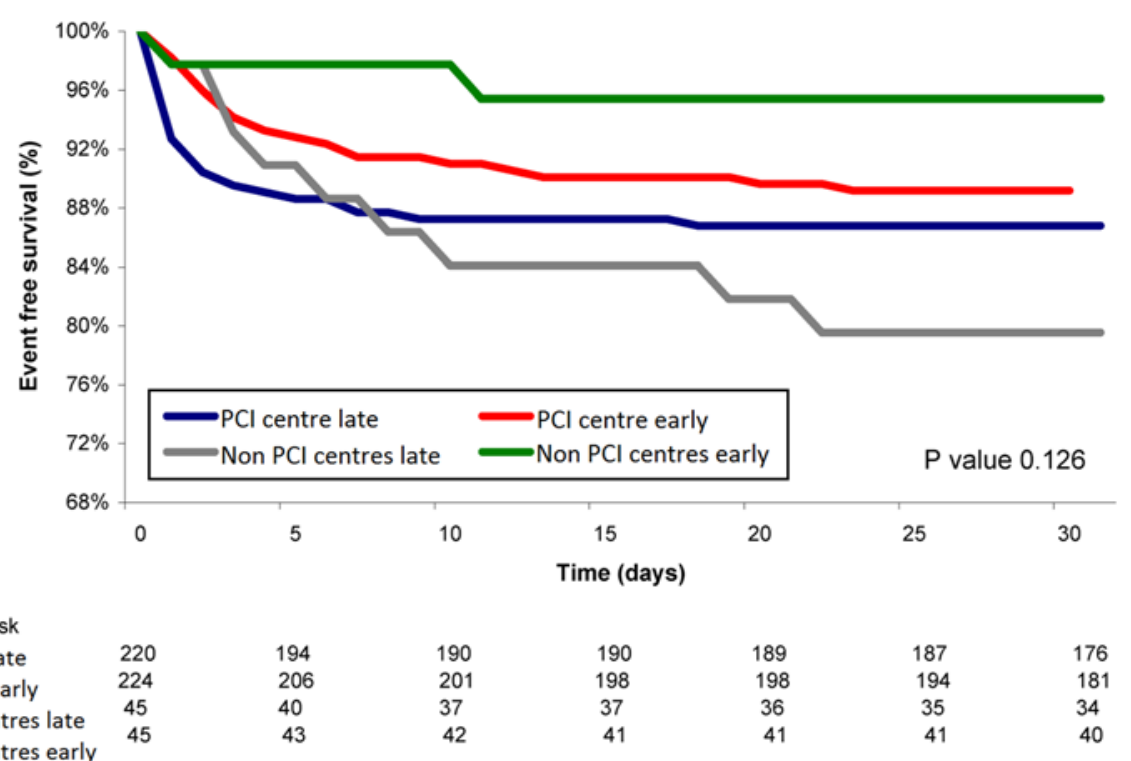

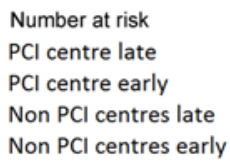

206
40
43
(RRR) for the combined endpoint of death, re-infarction or recurrent ischaemia at 30 days, driven by a reduction in recurrent ischaemia ( $\mathrm{RRR}=40 \%, p=0.058$ ). These results are largely consistent with previously conducted randomised trials and meta analyses [9-11] and show that immediate intervention is not associated with a reduction in hard clinical endpoints (death or myocardial infarction) but at most reduces the incidence of recurrent ischaemia. This post-hoc analysis shows that the sub-group of patients initially hospitalised in non-PCI centres especially benefit from an early invasive strategy. A plausible explanation is the shorter waiting time between angiography and revascularisation in these patients. 
Acknowledgments We would like to thank the staff in the coronary care units and the catheterisation laboratories and the cardiologists of the participating institutions. Furthermore, the aid with the statistical analysis of Evelien Kolkman and Petra Koopmans from Diagram (Diagnostic Research And Management- Zwolle, the Netherlands) was very much appreciated.

\section{Funding None.}

Conflict of interest None declared.

Open Access This article is distributed under the terms of the Creative Commons Attribution 4.0 International License (http://creativecommons.org/licenses/by/4.0/), which permits unrestricted use, distribution, and reproduction in any medium, provided you give appropriate credit to the original author(s) and the source, provide a link to the Creative Commons license, and indicate if changes were made.

\section{References}

1. Hamm CW, Bassand JP, Agewall S, et al. ESC Guidelines for the management of acute coronary syndromes in patients presenting without persistent ST-segment elevation: the Task Force for the management of acute coronary syndromes (ACS) in patients presenting without persistent ST-segment elevation of the European Society of Cardiology (ESC). Eur Heart J. 2011;32:2999-3054.

2. Task Force on Myocardial Revascularization of the European Society of Cardiology (ESC) and the European Association for Cardio-Thoracic Surgery (EACTS), European Association for Percutaneous Cardiovascular Interventions (EAPCI), Wijns W, Kolh P, Danchin N, et al. Guidelines on myocardial revascularization. Eur Heart J. 2010;31:2501-55.

3. Yan AT, Yan RT, Tan M, et al. In-hospital revascularization and one-year outcome of acute coronary syndrome patients stratified by the GRACE risk score. Am J Cardiol. 2005;96:913-6.

4. O'Donoghue M, Boden WE, Braunwald E, et al. Early invasive vs conservative treatment strategies in women and men with unstable angina and non-ST-segment elevation myocardial infarction: a meta-analysis. JAMA. 2008;300:71-80.

5. Mehta SR, Cannon CP, Fox KA, et al. Routine vs selective invasive strategies in patients with acute coronary syndromes: a collaborative meta-analysis of randomized trials. JAMA. 2005; 293:2908-17.

6. Lagerqvist B, Husted S, Kontny F, Stahle E, Swahn E, Wallentin L. 5-year outcomes in the FRISC-II randomised trial of an invasive versus a non-invasive strategy in non-ST-elevation acute coronary syndrome: a follow-up study. Lancet. 2006;368:998-1004.
7. Fox KA, Poole-Wilson P, Clayton TC, et al. 5-year outcome of an interventional strategy in non-ST-elevation acute coronary syndrome: the British Heart Foundation RITA 3 randomised trial. Lancet. 2005;366:914-20.

8. Fox KA, Clayton TC, Damman P, et al. Long-term outcome of a routine versus selective invasive strategy in patients with nonST-segment elevation acute coronary syndrome a meta-analysis of individual patient data. J Am Coll Cardiol. 2010;55:2435-45.

9. Bavry AA, Kumbhani DJ, Rassi AN, Bhatt DL, Askari AT. Benefit of early invasive therapy in acute coronary syndromes: a metaanalysis of contemporary randomized clinical trials. J Am Coll Cardiol. 2006;48:1319-25.

10. Damman P, Hirsch A, Windhausen F, Tijssen JG, de Winter RJ, ICTUS Investigators. 5-year clinical outcomes in the ICTUS (Invasive versus Conservative Treatment in Unstable coronary Syndromes) trial a randomized comparison of an early invasive versus selective invasive management in patients with non-STsegment elevation acute coronary syndrome. J Am Coll Cardiol. 2010;55:858-64.

11. Navarese EP, De Servi S, Gibson CM, et al. Early vs. delayed invasive strategy in patients with acute coronary syndromes without ST-segment elevation: a meta-analysis of randomized studies. QJM. 2011;104:193-200.

12. Katritsis DG, Siontis GC, Kastrati A, et al. Optimal timing of coronary angiography and potential intervention in non-ST-elevation acute coronary syndromes. Eur Heart J. 2011;32:32-40.

13. Navarese EP, Gurbel PA, Andreotti F, et al. Optimal timing of coronary invasive strategy in non-st-segment elevation acute coronary syndromes: a systematic review and meta-analysis. Ann Intern Med. 2013;158:261-70.

14. Van de Werf F, Gore JM, Avezum A, GRACE Investigators, et al. Access to catheterisation facilities in patients admitted with acute coronary syndrome: multinational registry study. BMJ. 2005;330:441-6.

15. Yusuf S, Flather M, Pogue J, et al. Variations between countries in invasive cardiac procedures and outcomes in patients with suspected unstable angina or myocardial infarction without initial ST elevation. OASIS (Organisation to Assess Strategies for Ischaemic Syndromes)Registry Investigators. Lancet. 1998;352:507-14.

16. Badings EA, The SHK, Dambrink JH, et al. Early or late intervention in high-risk non-ST-elevation acute coronary syndromes: results of the ELISA-3 trial. EuroIntervention. 2013;9:54-61.

17. IJkema BB, Bonnier JJ, Schoors D, Schalij MJ, Swenne CA. Role of the ECG in initial acute coronary syndrome triage: primary PCI regardless presence of ST elevation or non-ST elevation. Neth Heart J. 2014;22:484-90. 